\title{
Reflective Regression of 2D-3D Face Shape Across Large Pose
}

Xuhui Jia ${ }^{1}$

xhjia@cs.hku.hk

Heng Yang ${ }^{2}$

yanghengnudt@gmail.com

Xiaolong Zhu ${ }^{3}$

lucienzhu@gmail.com

Zhanghui Kuang ${ }^{4}$

kuangzhanghui@sensetime.com

Yifeng Niu²

niuyifeng@nudt.edu.cn

Kwok-Ping Chan ${ }^{1}$

kpchan@cs.hku.hk
${ }^{1}$ The University of Hong Kong

${ }^{2}$ National University of Defense Technology

${ }^{3}$ Tencent Inc.

${ }^{4}$ Sensetime Inc.

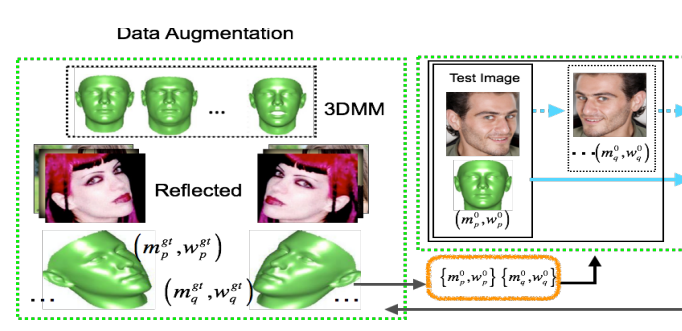

RCCR

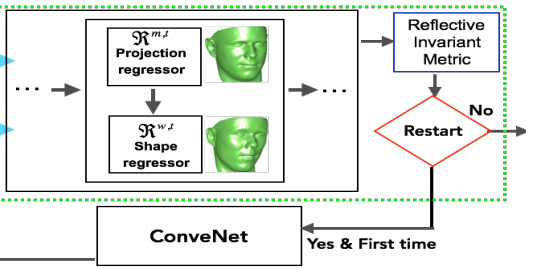

New initilisations can then be found by

In this paper we present a novel reflective method to estimate 2D-3D face shape across large pose. Based on the fact that 2D face image is a projection of 3D face model, we parameterise the configuration of landmarks into 3D Morphable Model and the projection matrix, and regress them in a unified framework. First, two regressors are learned for each cascaded stage, one for predicting the update of camera projection matrix, and the other for $3 \mathrm{D}$ shape parameters. They work collaboratively to refine the predicted shape towards true shape; Second, to tackle failures which always occur in largepose problem, we propose a novel reflective invariant metric to quantitatively estimate the alignments, subsequently the estimation will guide the model whether there is a need to restart the algorithm with different initialization. This is motivated by the fact that CPR are more sensitive to horizontal reflection, and the reflective variance are highly correlated to the misalignment error; Third, instead of using mean shape or random shapes for initialisation [1], we propose a head pose (from a ConveNet estimator) based initilisation scheme, which will relax failure alignments. searching samples with similar head pose in the training set. The main contributions of this paper are: 1) Large pose face alignment by fitting a dense 3DMM; 2) A novel reflective invariant metric, by investigating the relation between reflective variance and misalignment error; 3) A Reflective Cascaded Collaborative-Regressor algorithm that reduces large pose face alignment failures greatly.

In experiments, we evaluate the effectiveness of our proposed method in component-wise manner on AFLW test set. We compare to 1) RCCR without reflective feedback (CCR). 2) RCCR with reflective feedback and 5 random restart initialisations (RCCR). 3) RCCR with reflective feedback and 5 smart restart initialization (RCCR + SR). The comparison can be found in our paper, which shows, by using the reflective feedback, we achieve big improvement over CCR, which suggests us a failure-alarm mechanism is indeed very useful. Moreover, by using the head pose based initialisations, we achieve even better performance, though the improvement is relatively minor.

[1] Heng Yang and Ioannis Patras. Mirror, mirror on the wall, tell me, is the error small? In Proc. IEEE Conf. Comput. Vis. Pattern Recognit., 2015. 